\title{
Médiévales
}

Langues, Textes, Histoire

45 | automne 2003

Grammaires du vulgaire

\section{L'insuffisance des théories internes du changement phonétique : le cas de l'ancien français}

The Insufficiency of Internal Theories of Phonetical Change : the Case of Old French

\section{R. Anthony Lodge}

\section{OpenEdition}

Journals

Édition électronique

URL : https://journals.openedition.org/medievales/982

DOI : 10.4000/medievales.982

ISSN : $1777-5892$

Éditeur

Presses universitaires de Vincennes

Édition imprimée

Date de publication : 16 septembre 2003

Pagination : 55-66

ISBN : 2-84292-146-1

ISSN : 0751-2708

Référence électronique

R. Anthony Lodge, "L'insuffisance des théories internes du changement phonétique : le cas de

l'ancien français », Médiévales [En ligne], 45 | automne 2003, mis en ligne le 13 mars 2006, consulté le 23 avril 2022. URL : http://journals.openedition.org/medievales/982 ; DOI : https://doi.org/10.4000/ medievales.982

Ce document a été généré automatiquement le 23 avril 2022

Tous droits réservés 


\title{
L'insuffisance des théories internes du changement phonétique : le cas de l'ancien français
}

The Insufficiency of Internal Theories of Phonetical Change : the Case of Old French

\author{
R. Anthony Lodge
}

\section{NOTE DE L'ÉDITEUR}

Les cartes auxquelles réfère ce document sont présentes dans la version imprimée du numéro 45.

I Il a toujours existé au sein de la linguistique historique une tradition qui cherche à soustraire le déroulement des changements linguistiques au contrôle des personnes les plus directement concernées : les locuteurs. Pour les romantiques, la langue vit de sa vie propre, comme un arbre, dont la croissance est déterminée à la fois par le terrain et par des impulsions venues de sa composition interne. Pour les néogrammairiens la langue évolue suivant des lois phonétiques qui opèrent aveuglément, sans admettre d'exceptions. Pour les structuralistes, la langue est « un système où tout se tient ", un mécanisme dont les différentes parties synchronisent toutes seules leurs mouvements pour maintenir un équilibre interne d'oppositions et de contrastes. Même les dialectologues semblent partager cette vision de la vie autonome des langues, lorsqu'ils se servent de la métaphore anthropomorphique du "parler directeur» qui, dans une région donnée, montre la voie à des parlers qu'on devrait sans doute qualifier d'« exécutants». Envisager la langue comme un phénomène autonome comporte souvent des avantages réels, mais appliquer ce principe à l'explication du changement linguistique sans le considérer comme une pure fiction méthodologique risque de fausser considérablement notre vision des choses. 
2 La tradition normative place, certes, l'évolution de la langue sous la direction des hommes, mais non pas de tous les hommes, seulement celle d'une élite cultivée qui oriente soigneusement la langue dans le sens de la clarté et de l'élégance. À propos d'une prétendue «simplification » du français au XVII e siècle, W. von Wartburg estime qu'«un pareil développement ne peut pas partir des classes inférieures du peuple. Celui-ci n'a pas l'habitude de l'effort intellectuel. À une époque comme le xvII siècle, les forces directrices de la nation se concentrent dans les cercles des "honnêtes gens" $»^{1}$.

3 P. Fouché combine les deux approches: «Avant la Renaissance, on peut dire de l'évolution phonétique du français qu'elle était entièrement libre. Rien, dans l'ordre social, ne venant contrarier les forces de transformation [...] $»^{2}$. Au cours d'une longue période allant de la fin de l'Empire romain à la Renaissance, le changement linguistique en langue vulgaire aurait été gouverné uniquement par des lois internes. À partir du $\mathrm{XVI}^{\mathrm{e}}$ siècle, la situation change, le destin de la langue passerait à la classe instruite aux grammairiens, aux lexicographes, aux grands auteurs. Mais dans les deux cas, la communauté des locuteurs n'y serait pour rien.

4 Cette vision des choses repose à la fois sur une conception fragile du changement linguistique, et sur une représentation peu fiable de la société médiévale. En nous servant de données recueillies pour une étude de l'histoire sociolinguistique de Paris ${ }^{3}$, nous soutiendrons ici qu'il est impossible d'expliquer les changements phonétiques, même à l'époque médiévale, sans tenir compte de «l'ordre social». Ce sont les locuteurs qui font changer les langues et non pas l'inverse. En affirmant ceci, nous nous garderons bien, toutefois, d'attribuer à la communauté des locuteurs une intentionnalité quelconque : il s'agira nécessairement d'impulsions collectives venues de la « main invisible » invoquée récemment par R. Keller ${ }^{4}$.

Deux remarques préliminaires s'imposent : la première sur la difficulté de cerner les changements phonétiques dans les langues médiévales, la deuxième sur la distinction interne externe en matière de langue.

Le changement phonétique en ancien français

Pour des raisons qui se comprennent fort bien, les manuels destinés aux étudiants donnent une vision plus ou moins schématique des changements phonétiques en ancien français. Voici deux exemples : l'évolution du [e] long latin en syllabe tonique libre, et celle du suffixe latin [-ellos].

\begin{tabular}{|l|l|l|l|l|l|l|l|l|l|l|l|}
\hline Latin & $\mathrm{XI}^{\mathrm{e}}$ & $\mathrm{XII}^{\mathrm{e}}$ & $\mathrm{XIII}^{\mathrm{e}}$ & $\mathrm{XIV}^{\mathrm{e}}$ & $\mathrm{XV}^{\mathrm{e}}$ & $\mathrm{XVI}^{\mathrm{e}}$ & $\mathrm{XVII}^{\mathrm{e}}$ & $\mathrm{XVIII}^{\mathrm{e}}$ \\
\hline \hline $\mathrm{e}$ long & $\varepsilon \mathrm{j}$ & $\mathrm{Jj}$ & $\mathrm{w} \varepsilon$ & $>$ & $>$ & $>$ & $>$ & wa \\
\hline peram & & & & & & & & \\
\hline
\end{tabular}

\begin{tabular}{|l|l|l|l|l|l|l|l|l|l|l|}
\hline Latin & $\mathrm{XI}^{\mathrm{e}}$ & $\mathrm{XII}^{\mathrm{e}}$ & $\mathrm{XIII}^{\mathrm{e}}$ & $\mathrm{XIV}^{\mathrm{e}}$ & $\mathrm{XV}^{\mathrm{e}}$ & $\mathrm{XVI}^{\mathrm{e}}$ & $\mathrm{XVII}^{\mathrm{e}}$ & $\mathrm{XVIII}^{\mathrm{e}}$ \\
\hline \hline -ellos & ets & eaws & $>$ & $>$ & oos & วo & 0 & 0 \\
\hline sitellos & & & & & & & & seaux \\
\hline
\end{tabular}


7 De tels schémas ont une valeur pédagogique certaine, mais c'est au prix d'une simplification qu'on peut juger excessive. Bien que nous disposions de certains éléments - rimes, graphies et datations relatives - qui nous permettent de suivre l'évolution progressive des sons, dans une langue comme l'ancien français (qui n'est que la somme de ses composantes dialectales), qu'est-ce qui nous permet de préciser le moment où tel ou tel changement phonétique serait arrivé effectivement à terme?

Les prescriptivistes prennent comme critère l'acceptation de l'innovation par la norme. Ce critère peut, à la rigueur, fonctionner dans les langues standardisées du monde moderne, mais il est difficile de l'appliquer au français non-standardisé du Moyen Âge. Dans ce dernier cas, la solution souvent retenue est donc d'inventer une langue standard médiévale, baptisée en l'occurrence le «francien ", qui existe avant tout pour entériner les changements phonétiques et morphologiques successifs. Les diachroniciens structuralistes ont eux aussi leurs raisons pour affectionner une langue médiévale standardisée. Pour eux, l'étude du changement linguistique consiste à confronter des coupes synchroniques pratiquées à différents stades de la diachronie. Il convient, à chaque stade, d'idéaliser une variété particulière, de la dépouiller autant que possible de variations, afin de faire ressortir le système sous-jacent d'oppositions et de contrastes phonologiques.

9 Ce qui se trouve gommé dans ces deux types d'analyses, c'est le caractère continu et inhérent du changement linguistique et le rapport indissociable entre changement et variation. Comme l'a bien vu A. Meillet au début du vingtième siècle, une langue vivante voit constamment naître des innovations (des variations), et ces innovations sont constamment en train de se propager à travers la langue d'une part (la diffusion lexicale) et à travers la société d'autre part ${ }^{5}$. Le changement s'imbrique dans la variation, comme la variation dans la communauté des locuteurs.

Les distinctions externe $\sim$ interne, exogène $\sim$ endogène

10 La distinction externe interne remonte, en linguistique, au moins jusqu'à Saussure. Tandis que l'histoire interne se focalise sur l'évolution du système de la langue, en faisant abstraction des locuteurs, l'histoire externe concerne les circonstances économiques, démographiques, politiques des locuteurs et les forces sociales auxquelles ceux-ci sont exposés. Bien que la deuxième donne le cadre à la première, il est de tradition de mener les deux histoires séparément : « La langue est un système qui ne connaît que son ordre propre $»^{6}$. Or, la sociolinguistique historique récente met cette ségrégation sérieusement en cause : nous allons voir que la structuration « externe » du groupe de locuteurs peut peser lourdement sur l'évolution « interne » de leur langue 7 .

11 Une deuxième distinction rejoint cette première, sans pourtant la recouper, c'est celle entre innovations endogènes et innovations exogènes. Tandis que les changements endogènes trouvent leur origine à l'intérieur du groupe parlant la langue/dialecte en question, les changements exogènes sont le résultat de contacts avec des groupes parlant d'autres langues/dialectes. Quand il s'agit du français du Moyen Âge, l'historiographie traditionnelle privilégie presque toujours le premier au détriment du second, les mélanges de langues et de dialectes étant normalement vus avec une certaine répugnance (ce seraient autant de cas d'hybridation et d'abâtardissement de la langue).

Dans ce qui suit, nous allons prendre le cas du français de Paris au Moyen Âge pour montrer, d'une part, comment des facteurs externes (le contexte démographique de la 
ville) ont pu exercer un effet déterminant sur l'évolution linguistique interne; et, d'autre part, comment les deux exemples de changements phonétiques cités plus haut se comprennent mieux dans la langue de la métropole, si l'on suppose une origine exogène plutôt qu'endogène.

Facteurs externes contre facteurs internes : le cas de Paris

$C^{\prime}$ 'est seulement avec de très grandes précautions que l'on viendra s'attaquer à l'immense contribution d'A. Martinet à notre compréhension du déroulement des changements phonétiques ${ }^{8}$. Nous nous bornerons à soutenir ici que le jeu des facteurs internes, si admirablement exposé par lui, est toujours à compléter, néanmoins, par l'effet de facteurs sociaux. Avec U. Weinreich, W. Labov et M. Herzog' ${ }^{9}$, nous sommes en droit de poser la question de savoir, à propos de tout changement linguistique, pourquoi à cet endroit et à ce moment-là de l'histoire, et non pas à tel autre endroit et à tel ou tel autre moment ? En fait, la variation « libre » de la linguistique structuraliste s'avère rarement libre. Elle est conditionnée la plupart du temps par les évaluations subjectives des locuteurs face aux variantes en présence. Les représentations des locuteurs se répercutent d'abord dans les pratiques linguistiques, pour en venir, plus tard, à affecter le système lui-même.

Une des découvertes les plus intéressantes de la sociolinguistique historique récente est la révélation des effets qu'exerce la structure du groupe sur la structure de la langue. H. Andersen a comparé le développement de dialectes situés en zone centrale (comme ceux des grandes métropoles) et celui de dialectes situés en zone périphérique ${ }^{10}$. Les notions de "centre » et de " périphérie » portent moins sur la distance géographique que sur la densité et l'orientation de réseaux d'interaction. Il constate que les communautés linguistiques situées en zone centrale connaissent des taux de communication inter-dialectale relativement élevés, ce qui leur vaut l'étiquette de "dialectes ouverts", et que les groupes situés en périphérie connaissent un taux de communication inter-dialectale inférieur, ce qui leur attire le titre de «dialectes fermés ». H. Andersen fait une deuxième distinction, qui peut ou non être surimposée à la première, entre "communautés dialectales exocentriques" et "communautés dialectales endocentriques ». Il s'agit ici des attitudes subjectives des locuteurs envers leur dialecte, les communautés exocentriques étant plus prêtes à admettre l'influence de comportements linguistiques extérieurs, les communautés endocentriques cherchant à conserver et à défendre plus vigoureusement leurs normes linguistiques ancestrales.

$\mathrm{H}$. Andersen constate que les dialectes « ouverts » et « exocentriques » connaissent des changements linguistiques plus rapides et qu'ils comportent des systèmes phonologiques et morphologiques relativement plus "simples", tandis que les dialectes "fermés» et «endocentriques" font l'inverse: ils ont des systèmes phonologiques et morphologiques conservateurs et relativement complexes. Il y une affinité évidente entre ces idées et celles de R. Le Page et A. Tabouret-Keller sur les actes d'identité ${ }^{11}$, ainsi qu'avec celles de L. Milroys sur les réseaux sociaux ${ }^{12}$. La réussite d'une innovation linguistique est déterminée autant par sa capacité à symboliser les distinctions sociales que par ses propriétés linguistiques internes. Pour A. TabouretKeller, les communautés fortement focalisées sont souvent caractérisées par une conscience très développée des normes linguistiques. Pour L. et J. Milroy, la présence au sein du groupe de réseaux sociaux faibles a pour effet d'accélérer le processus de changement linguistique, tandis que la présence de réseaux sociaux denses a l'effet inverse $^{13}$. 

au dix-septième siècle le parler urbain de Paris et le parler rural des paysans vivant en Île-de-France, à cinq ou six kilomètres seulement de Notre-Dame. Voici un extrait d'un texte très connu où l'auteur cherche à imiter le langage des paysans de la banlieue parisienne, au moment de la Fronde :

Piarot:

Hé ban n'importe cest une tache douile, ça sen va à la leuscivre. Mai say tu ban que je revinme jesque dan nout vilage : bon jou bon soir, je ne sçay san que tu devins, je m'en alli sous l'orme, où je trouvi nout vilage amassé qui m'attendien pour var le zerticle de la Paix. Dé que je fu venu, nan lé baiy à luise à Colin, qui lui queme un Ange, quer y chante l'Eupitre queme un enragé, quan y leust luy tou depi un bou jesqu'a l'outre, vla tou le mondre qui se boutte a marmuze : voize diset lun, vla ban opezé, je some ban planté pour rvardi, nou vla tretou oussi gras que de liau; queman sdiset loutre, ne vlati pas la taye à cu, morgué je nan poigeron poen ce quarquié cy ; ban entandu slidije, quer jay beu l'argen de mon viau, je nan poigeray poen; ty es laisti chouar sdit le receveux, stu nas poen doutre chifflet ton chian est pardu, nan ne tan rabattra pas une oborle, lui ce placart tou ton sou, si tu li trouve je veux que nan me pele la berbe. ${ }^{14}$

Certains érudits ont été tellement déroutés par le caractère bizarre des formes contenues dans des textes comme celui-ci, qu'ils ont été portés à croire qu'il s'agissait de formes entièrement factices, que les nombreuses attestations que nous possédons du dialecte traditionnel de l'île-de-France aux $\mathrm{XVII}^{\mathrm{e}}$-XVIII ${ }^{\mathrm{e}}$ siècles sont autant de fabrications littéraires ${ }^{15}$. Nul ne doit négliger le caractère stéréotypé de ces représentations du parler rural, mais remplacer une foi naïve dans ces documents par un scepticisme total est tout aussi déplacé.

L'écart qui séparait au $\mathrm{XvII}^{\mathrm{e}}$ siècle le parler de la ville de celui de la campagne environnante s'explique parfaitement bien si l'on tient compte du fait que le parler des villes évolue toujours plus vite. Dès le XIII ${ }^{e}$ siècle, Paris est devenue la plus grande concentration d'hommes de l'Occident, le lieu où le taux d'interactions et de communications inter-dialectales était plus élevé que partout ailleurs. Il n'est nullement surprenant dans ces circonstances que le parler de Paris ait commencé, depuis ce moment-là, à s'élever progressivement au-dessus du continuum dialectal du reste du domaine d'oïl. Une conscience de l'écart entre le parler intra muros et le parler extra muros est attestée à Paris déjà au $\mathrm{Xv}^{\mathrm{e}}$ siècle $^{16}$. On peut même se demander si, au XIII ${ }^{\mathrm{e}}$ siècle, Roger Bacon ne faisait pas déjà une distinction entre "francien " et « français de Paris » quand il affirme que « picard, normand, bourguignon, parisien et français : ce n'est qu'une seule et même langue, à savoir le français; mais elle varie accidentellement dans différentes régions; cette diversité en fait des dialectes différents et non des langues différentes $»^{17}$.

Pour bien comprendre le déroulement des changements phonétiques à Paris et en Îlede-France au Moyen Âge, il est indispensable de replacer ces processus dans la communauté des locuteurs: des facteurs «externes» tels que la mobilité démographique, la nature des réseaux sociaux, les taux d'interaction ont eu un impact considérable sur la rapidité et sur la direction des changements linguistiques « internes ». L'effet en a été l'éloignement du parler « progressif » de la ville des parlers plus « conservateurs » des campagnes environnantes.

Changements exogènes contre changements endogènes 

langues/dialectes en contact, notamment dans la théorie des «strats " (substrat, superstrat etc.). On constate cependant dans l'histoire du français que l'influence des " strats » se cantonne généralement dans des périodes très anciennes, soit juste avant, soit juste après la période romaine. L'époque médiévale n'en est guère affectée. S'agissant d'une société foncièrement rurale et statique, où les contacts entre les régions sont sévèrement limités et où les villes, Paris y compris, n'ont qu'un rôle secondaire à jouer, les interférences venues d'autres dialectes ou d'autres langues (en dehors du latin) sont très réduites : les innovations linguistiques sont de nature surtout endogène, permettant à la langue d'évoluer «librement» (selon l'expression de P. Fouché), c'est-à-dire suivant les seules lois phonétiques.

作 linguistes ont fait grand cas de la pureté linguistique, culturelle et même raciale. Les mélanges de langues et de dialectes donnaient naissance à des langues hybrides qui pouvaient convenir à des races d'esclaves comme les langues créoles, mais qui étaient tout de même indignes d'une grande nation. F. Brunot était conscient du caractère mixte du "francien", mais, visiblement, cela le gênait, car il éprouve le besoin d'affirmer aussitôt que « le francien ne doit pas être considéré comme un amalgame $»^{18}$.

Cette vision statique rurale de la civilisation française au Moyen Âge est aujourd'hui rejetée en tous points : ce qui a permis l'éclosion de cette civilisation au XIII ${ }^{\mathrm{e}}$ siècle, c'est précisément la mobilité des hommes et l'essor des villes, à commencer par Paris. Promu au rang de première ville d'Europe, Paris devient au xiII ${ }^{\mathrm{e}}$ siècle un pôle d'une importance primordiale. Sa population de 200000 habitants (immense pour l'époque) est alimentée presque entièrement par l'immigration, de l'île-de-France d'abord, de Normandie et de Champagne ensuite. Les historiens de la langue ne peuvent pas ne pas prendre en considération des évolutions démographiques et économiques de cette ampleur. Dès le XIII ${ }^{\mathrm{e}}$ siècle, Paris est devenue le grand creuset dialectal de la GalloRomania.

On peut dire du système phonologique et morphologique de base du français parisien qu'il est resté proche de celui des dialectes de l'île-de-France, mais il convient d'ajouter qu'il a été modifié d'une manière significative par l'incorporation de nombreux éléments exogènes ${ }^{19}$. Prenons les deux exemples cités plus haut de changements phonétiques dans le français parisien du Moyen Âge. Ceux-ci s'expliquent mal par le seul jeu des facteurs endogènes et internes :

(i) $[\varepsilon]>[\mathrm{ej}]>[\mathrm{j}]]>[\mathrm{we}]>[\mathrm{wa}]$

(ii) $[$-ellos $]>[$-eaus $>>$ [jo] $/$ [əo] $>[$ o]

Pour bien comprendre ces évolutions, le témoignage de certaines cartes de l'Atlas Linguistique de la France (ALF) est un outil instructif (car, jusqu'à un certain point, le passé fait toujours partie de notre présent - s'il est légitime de considérer que le début $\mathrm{du} \mathrm{xx}^{\mathrm{e}}$ siècle soit encore du présent). Il sera exclu que les deux cartes qui vont suivre nous donneront la configuration des isoglosses au Moyen Âge. Bien au contraire, ces 
cartes impliquent précisément des mouvements de formes depuis cette époque reculée. Mais dans quel sens, Province $>$ Paris ou Paris $>$ Province?

[we] [wa]

L'évolution [e] (tonique libre) > [ej] > [we] > [wa] (par ex. roi, mois, poids), entre le XIII et le $\mathrm{xvIII}^{\mathrm{e}}$ siècle, et la diffusion sociale et lexicale de la variante [wa] ont fait l'objet d'analyses minutieuses ${ }^{20}$. Cependant, l'incertitude plane toujours sur les origines de cette innovation. Certains soutiennent qu'il s'agit d'un changement endogène, que l'ouverture de [we] en [wa] est conforme à une habitude répandue dans le français parisien d'ouvrir $[\varepsilon]$ en [a], surtout devant [r] (par ex, Pierrot > Piarot]. Mais une explication exogène à ce changement est au moins aussi plausible.

Le [ê/1̄] latin accentué s'est diphtongué en [ej] dans tout le nord gallo-roman à une époque très ancienne. On peut voir les évolutions ultérieures sur la carte $\mathrm{n}^{\circ} 1$ tirée de l'ALF :

Carte 1 - poire (ALF 1047)

Tandis que dans l'ouest cette diphtongue se réduit à $[\varepsilon]$, dans le centre elle passe à [wE], et dans l'est à [wa]. Ce qui m'intéresse dans cette configuration, c'est la petite saillie de formes en [wa] à l'ouest de Paris. Elle suggère que [wa] s'est développée d'abord dans les dialectes de l'est, qu'elle s'est répandue petit à petit à Paris avant d'être diffusée à une époque récente dans les dialectes de la grande banlieue. On peut imaginer que la variante [wa], attestée sporadiquement dans le français de Paris dès le $\mathrm{XIII}^{\mathrm{e}}$ siècle ait eu une origine endogène, et que, par la suite, elle se soit diffusée en priorité vers les dialectes de l'est. Mais une explication comportant une origine exogène (dialectes de l'est > Paris) serait nettement plus simple.

$$
\text { [jo] [o] }
$$

L'exemple de la variable [o] [jo] se laisse expliquer de façon analogue. La vocalisation de [1] devant [s] dans le groupe -els (< Latin -ellos) a entraîné l'insertion d'une voyelle [a] de transition et la formation d'une triphtongue [eaw]. L'évolution de cette triphtongue a été différente selon les régions :

Carte 2 - manteau (ALF 812)

Dans la plus grande partie du domaine d'oïl le premier élément de la triphtongue s'est consonantisé en [j] :

$$
\text { [-ellos }]>[\text {-eaus }]>[\text {-jaws }]>[- \text { jos }]>[- \text { jo }]
$$

Mais dans la région parisienne, comme dans une zone située à l'est de la métropole, le premier élément passe à [ə] avant d'être absorbé par les éléments vocaliques suivants :

[-ellos $]>[$-eaus $]>[$-əaws $]>[$-əos $]>[$-o $]$

Entre le XIV et le XIX ${ }^{e}$ siècles les deux variantes se concurrencent dans le parler parisien (un seau d'eau versus un siau d'iau) avant la disparition des formes en [jo]. Ceci dit, les rapports entre les deux variantes dans le français de Paris sont encore loin de faire l'unanimité des observateurs. Quelle serait la forme endogène?

Pour ce qui est de la chronologie, A. Dauzat considère que la variante $[0]<[ə o]$ est la première en place à Paris, et que [jo] est une forme rustique arrivée plus tard, qui n'a jamais réellement pénétré la langue de la capitale ${ }^{21}$. L'étude des graphies dans les documents parisiens du Moyen Âge ne lui donne pas raison. Nous avons examiné les 
archives de la Prévôté de Paris du milieu du XIII au milieu du XIV ${ }^{\mathrm{e}}$ siècle, rassemblées électroniquement par P. van Reenen. Pour le XIII ${ }^{\mathrm{e}}$ siècle, la situation est claire et nette : la graphie « eau » n'est attestée nulle part. C'est «iau » partout. Qu'en est-il au xiv siècle? Les résultats se trouvent dans le tableau $\mathrm{n}^{\circ} 1$ (les chiffres placés entre parenthèses indiquent le nombre d'occurrences de la forme en question).

TAB. 1 - LA VARIABLE « -IAU » " -EAU » DANS LES ARCHIVES DE LA PRÉVÔTÉ DE PARIS (xiv SIÈCLE)

\begin{tabular}{|c|c|c|}
\hline & -iau & -eau \\
\hline 1300-1319 & $\begin{array}{l}\text { Boifliaue (1) } \\
\text { Jumiaus (6) } \\
\text { yaues (1) }\end{array}$ & - \\
\hline $1320-1339$ & $\begin{array}{l}\text { appiaus (16) } \\
\text { biau (1) } \\
\text { Guibonniau (1) } \\
\text { hiaumer (1) } \\
\text { iaue (1) } \\
\text { nouviau (3) } \\
\text { tonniau (4) } \\
\text { trumiaus (1) } \\
\text { Ysabiau (1) }\end{array}$ & heaumier (1) \\
\hline $1340-1365$ & $\begin{array}{l}\text { aniaux (1) } \\
\text { appiaus (5) } \\
\text { Biauvez (2) } \\
\text { Blondiau (6) } \\
\text { boissiau (1) } \\
\text { Charonniau (1) } \\
\text { Grimardiau (1) } \\
\text { quarriaus (1) } \\
\text { Roussiau (1) } \\
\text { Ysabiau (5) }\end{array}$ & $\begin{array}{l}\text { appeaulx (1) } \\
\text { Beaufort (1) } \\
\text { Boileau (1) } \\
\text { eaues (1) } \\
\text { nouveau (1) }\end{array}$ \\
\hline
\end{tabular}

La graphie « eau » fait sa première apparition dans les Archives de la Prévôté durant le deuxième quart $\mathrm{du} \mathrm{xIV}^{\mathrm{e}}$ siècle seulement, mais «iau» reste largement majoritaire jusqu'à la fin du siècle. Visiblement, le tard-venu sur la scène parisienne, c'est la graphie " eau ", non « iau ». Dès le milieu du siècle suivant, la variante « iau » disparaît complètement de l'orthographe parisienne. Quant à la prononciation [jo], quoique bannie très tôt du bon usage, celle-ci persiste longtemps dans l'usage vernaculaire parisien, d'où elle ne disparaît qu'au cours du Xix ${ }^{e}$ siècle.

Mais qu'en est-il des origines de la variante [o]? Certains observateurs ne se prononcent pas sur la question de ses origines ${ }^{22}$. La majorité lui attribue une origine 
endogène ${ }^{23}$, en dépit du témoignage des graphies médiévales. Quel est l'apport des données cartographiques contenues dans l'Atlas Linguistique de la France (voir carte $\mathrm{n}^{\circ}$ 2) ? La concentration de formes monophtonguées dans une région située à l'est de Paris et la présence d'une petite saillie dans la banlieue ouest nous oriente, comme dans le cas de [wa], vers une origine exogène de la variante [o], la forme [jo] étant la forme endogène.

Le but de cette étude n'est pas de remplacer l'approche structurale interne des changements phonétiques par une approche sociolinguistique purement externe. On a besoin des deux approches, car elles influent réciproquement l'une sur l'autre: les changements phonétiques sont dus aux locuteurs, mais se manifestent tôt ou tard dans le système interne de la langue ${ }^{24}$. Cela n'empêche toutefois que pendant trop longtemps on a considéré qu'il était possible de traiter le problème du changement linguistique en ancien français dans un vide social. On n'a pas tenu suffisamment compte des effets que peut exercer la structure du groupe sur la structure interne de la langue. On a trop longtemps sous-estimé la mobilité de la population française au Moyen Âge et le rôle central de Paris dans la migration des formes et dans le mélange des dialectes. Ce sont les locuteurs qui font évoluer les langues et non l'inverse, sans le savoir et sans le vouloir, bien entendu ${ }^{25}$.

\section{NOTES}

1. W. von Wartburg, Évolution et structure de la langue française, Berne, 1962, p. 176.

2. P. Fouché, «L'évolution phonétique du français du xvie siècle à nos jours », Le Français Moderne, 2, 1934, p. 218.

3. R. A. LodGE, A Sociolinguistic History of Parisian French, Cambridge, 2003.

4. R. KeLLER, On Language Change. The Invisible Hand in Language, Londres, 1994.

5. A. Meillet, « Comment les mots changent de sens », dans Linguistique historique et linguistique générale, Paris, 1912, p. 230-271.

6. F. de Saussure, Cours de linguistique générale, Paris, 1916, p. 40-43.

7. Voir en particulier W. Labov, Principles of Linguistic Change, t. 1, Internal Factors, Oxford, 1994, p. 300-301.

8. A. Martinet, $L$ 'Économie des changements linguistiques, Berne, 1955.

9. U. WeinReich, W. LABOV, M. HERZOG, « Empirical foundations for a theory of language change ", dans W. P. LehMANN et Y. MALKIEL éd., Directions for Historical Linguistics, Austin, 1968, p. 95-195.

10. H. ANDERSEN, « Centre and periphery : adoption, diffusion and spread », dans

J. FISIAK éd., Historical Dialectology. Regional and Social, Berlin/New York/Amsterdam, 1988, p. 39-83.

11. R. Le Page et A. Tabouret-Keller, Acts of Identity, Cambridge, 1985.

12. J. MiLROY, Linguistic Variation and Change, Oxford, 1992.

13. "Relationships in tribal societies, villages and traditional working-class communities are typically multiplex and dense, whereas those in geographically and 
socially mobile industrial societies tend to uniplexity and spareness » (L. MiLRoY, Language and Social Networks, Oxford, 1987, p. 52). « Linguistic change is slow to the extent that the relevant populations are well-established and bound by strong ties ; whereas it is rapid to the extent that weak ties exist in populations » (J. et L. MiLROY, " Linguistic change, social network and speaker innovation ", Journal of Linguistics, 21, 1985, p. 375).

14. Agréables Conférences de deux paysans de Saint-Ouen et de Montmorency sur les affaires du temps (1649-1651), J. Deloffre éd., Paris, 1999, p. 126-127.

15. G. Bergounioux, « Le francien (1815-1914) : la linguistique au service de la patrie », dans Les Langages du politique, Mots, 19, 1989, p. 23-40.

16. Voir la Farce des Enfants de Bagneux, dans Recueil de farces françaises inédites $d u X^{e}$ siècle, G. Cohen éd., Cambridge (Mass.), 1949.

17. «Ut Picardum et Normanicum, Burgundicum, Parisiense et Gallicum : una enim lingua, est omnia, scilicet Gallicana, sed tamen in diversis partibus diversificatur accidentaliter ; quae diversitas facit idiomata non linguas diversas » (Roger Bacon, Compendium studii philosophiae, Londres, 1859, t. VI, p. 478-9).

18. F. Brunot, Histoire de la langue française, Paris, t. 1, p. 325.

19. C. Fondet, La Dialectologie de l'Essonne. Paris, 1980, et "Contribution à la question des origines du français : quelques aperçus à partir de la dialectologie de l'Essonne », dans M. Tamine éd., Ces Mots qui sont nos mots. Mélanges d'histoire de la langue francaise, de dialectologie et d'onomastique offerts au professeur Jacques Chaurand, Charleville-Mézières, 1995, p. 189-206.

20. Cf. J. REIGHARD, « The transition problem : lexical diffusion vs. variable rules », dans Papers from the 4th International Conference on Historical Linguistics, E. C. TRAUGOTT, R. LABRUM, S. SHEPHERD éd., Current Issues in Linguistic Theory, 14, 1980, p. 349-354.

21. A. Dauzat, Les Patois, Paris, 1927, p. 40.

22. F. de la Chaussée, Initiation à la phonétique historique de l'ancien français, Paris, 1974, p. 116, et J. Picoche et C. Marchello-Nizia, Histoire de la langue française, $4^{\mathrm{e}}$ édition, Paris, 1994, p. 194.

23. M. K. Pope (From Latin to Modern French, Manchester, 1952, § 540) et E. Bourciez (Phonétique française, Paris, 1967, § 48) considèrent, à la suite de Dauzat, que « -eau » est une forme d'origine parisienne. P. Fouché (Phonétique historique du français, Paris, 1969, p. 336) voit [o] < [eo] comme une variante aristocratique créée pour distinguer la classe supérieure du reste de la population. J. Wüest (" Le patois de Paris et l'histoire du français », Vox Romanica, 44, 1985, p. 243) le suit dans cette voie, affirmant que « la différence entre eau et iau ne pouvait donc être dialectale; elle devait être sociolectale ». G. Zink (Phonétique historique du français, Paris, 1986, p. 141) y voit une opposition " populaire - savant », sans dire pourtant ce que « -eau » a de savant ou de livresque.

24. Voir J. MiLroy, Linguistic Variation and Change, op. cit., p. 280.

25. Je tiens à remercier Madame $\mathrm{C}$. Marchello-Nizia qui a eu l'obligeance de corriger mon texte avant qu'il ne soit soumis aux éditeurs. 


\section{RÉSUMÉS}

On a trop longtemps situé le problème du changement phonétique en ancien français dans un vide sociolinguistique, où les facteurs sociaux étaient censés n'avoir qu'un impact minimal sur les structures linguistiques internes, et où une société médiévale, avant tout rurale et statique, inhibait les contacts entre les dialectes et les migrations de formes linguistiques. L'objet de cette étude n'est pas de remplacer les explications internes, structurales, par des explications purement externes et sociolinguistiques, mais d'illustrer la nécessité d'imbriquer le changement linguistique dans la communauté des locuteurs. En prenant deux cas de figure tirés de l'ancien français parisien (les évolutions $[\mathrm{e}]>[\varepsilon j]>[\mathrm{w} \varepsilon]>[\mathrm{wa}]$, et -ellos $>$ [eaws] / [jaws] $>$ [o]), nous chercherons à montrer que de tels changements ne peuvent pas être compris en invoquant uniquement des facteurs internes, et que des facteurs sociaux, tels les niveaux d'interaction sociale et le mélange de dialectes dans la grande ville, ont joué un rôle décisif.

The Insufficiency of Internal Theories of Phonetical Change : the Case of Old French. For too long, sound change in Old French has been considered in a sociolinguistic vacuum, where social factors are deemed to have no impact on internal linguistic structure, and where medieval society is seen as overwhelmingly rural and static, inhibiting serious levels of dialect contact and the migration of linguistic forms. The purpose of this paper is not to replace internal, structural explanations of sound change with a purely external, sociolinguistic one, but to illustrate the need to embed language change permanently within the community of speakers. Taking as examples two cases of phonetic change in medieval Parisian French $([\mathrm{e}]>[\varepsilon \mathrm{j}]>[\mathrm{w} \varepsilon]>[\mathrm{wa}]$ and -ellos $>$ [eaws] / [jaws] $>[o]$ ), the paper shows that sound-changes such as these cannot be understood on the basis of language-internal pressures alone, and that levels of social interaction and dialect-mixing have a decisive role to play.

\section{INDEX}

Mots-clés : ancien français, phonétique, sociolinguistique, changements linguistiques, dialectes Keywords : Old French, phonetics, sociolinguistics, linguistic change, dialects

\section{AUTEUR}

\section{R. ANTHONY LODGE}

Department of French, University of St Andrews, Fife, G-B - KY16 9PH, anthony.lodge@stand.ac.uk 